\title{
Bilateral obstructive uropathy following laparoscopic appendicectomy.
}

\author{
Nathan Grimes $^{1 *}$, Sean McMahon ${ }^{2}$, Ajay Pahuja ${ }^{3}$, Hugh O'Kane ${ }^{3}$ \\ ${ }^{1}$ Department of Urology, Forth Valley Royal Hospital, Larbert, Scotland \\ ${ }^{2}$ Queen's University Belfast, Belfast, Northern Ireland \\ ${ }^{3}$ Department of Urology, Belfast City Hospital, Belfast, Northern Ireland
}

\begin{abstract}
A fifty four year old man presented with renal failure several days following laparoscopic appendicectomy. Imaging revealed a large rectus sheath haematoma extending into the pelvis causing bilateral ureteric obstruction. The initial reason for renal failure was not clear as imaging did not reveal significant hydronephrosis as would usually be expected with ureteric obstruction, however renal function failed to improve despite addressing pre-renal causes of renal impairment. He was treated with both a retrograde ureteric stent and percutaneous nephrostomy.
\end{abstract}

\section{Introduction}

Treatment of obstructive uropathy is a common urological referral. The two treatment options are nephrostomy tube or ureteric stent insertion. Both are equally effective at providing collecting system drainage and as both have different specific advantages and disadvantages, choice is often made on a case-by-case basis [1]. Percutaneous nephrostomy has the advantages of bypassing the level of ureteric obstruction and not requiring a general anaesthetic, which can sometimes lead to deterioration in an already acutely unwell patient. The procedure can be technically difficulty in overweight patients, and those without hydronephrosis, and is not without risk of significant haemorrhage. Retrograde uretericstent insertion has a lower rate of significant bleeding than percutaneous nephrostomy insertion; however it is sometimes not possible to gain access to the kidney, such as in cases of large impacted stones or abnormal urinary tract anatomy due to pelvic disease. Here, we present a case with a rare cause of bilateral ureteric obstruction secondary to an iatrogenic pelvic haematoma.

\section{Case Report}

A fifty four year old male, with a metallic aortic valve on warfarin, presented with acute appendicitis. He underwent laparoscopic appendicectomy which revealed a necrotic appendix. Initial post-operative recovery was uneventful and he was discharged seven days following admission.

He re-presented three days after discharge with hypovolaemic shock and abdominal distension. CT abdomen/ pelvis showed rectus sheath haematoma with significant abdominal and pelvic extension (Figures 1 and 2). His warfarin was reversed and a bleeding inferior epigastric artery was controlled by embolisation by interventional radiology.

Over the subsequent days, he developed progressive renal impairment, with creatinine rising from a normal baseline on

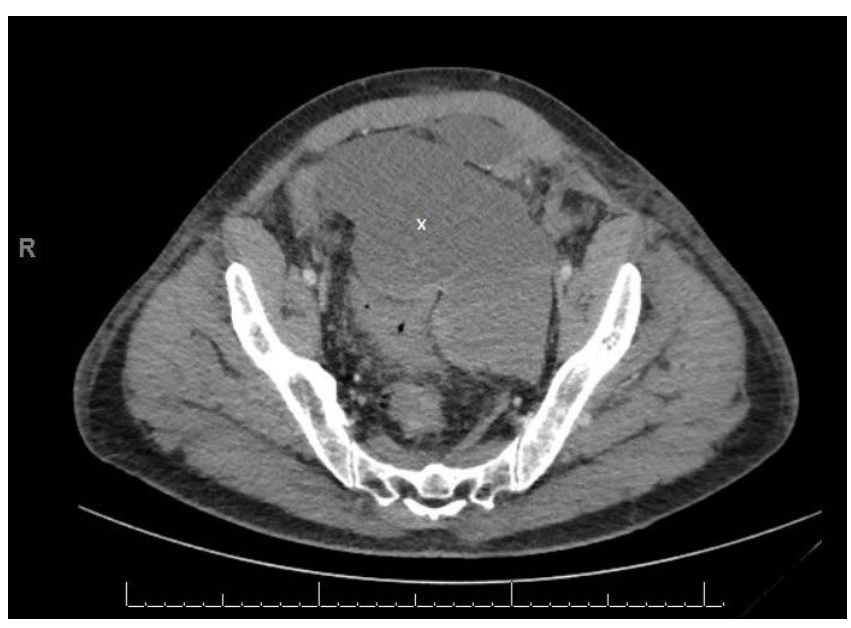

Figure 1. CT demonstrating large rectus sheet haematoma extending into pelvis marked with " $x$ ".

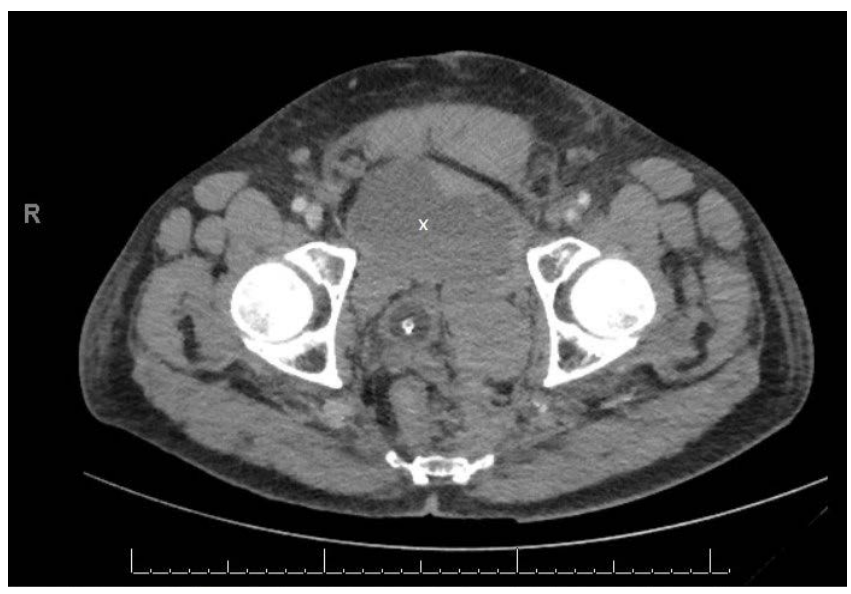

Figure 2. CT demonstrating pelvis (marked with " $x$ ”) distorting catheterized bladder.

admission to 270 despite haemorrhage control, transfusion of packed red cells and adequate intravenous fluid resuscitation. 


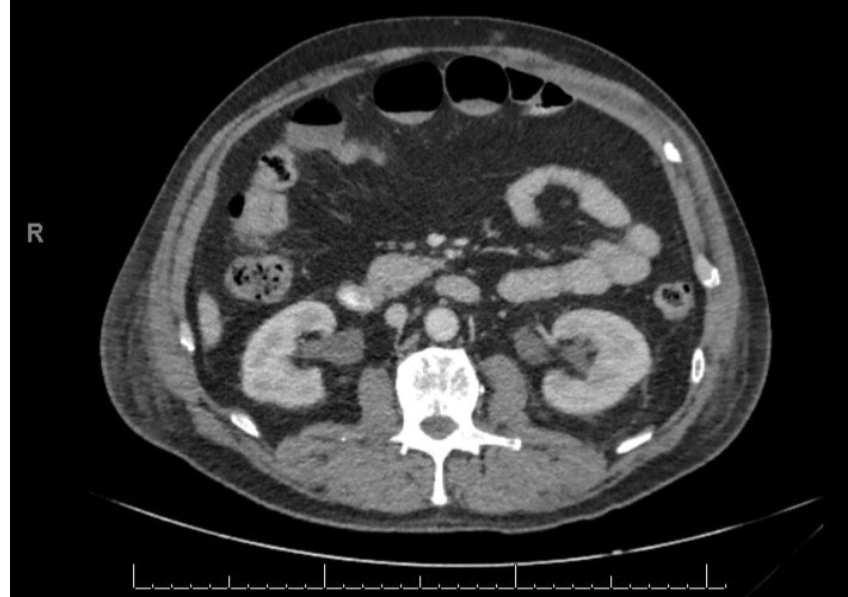

Figure 3. CT demonstrating lack of hydronephrosis despite obstruction.

Repeat imaging revealed minimal fullness of his right renal collecting system and normal left collecting system (Figure 3).

Cystoscopy revealed a grossly distorted bladder due to extrinsic compression by the haematoma. The right ureteric orifice was identified and a right ureteric stent was placed day one of re admission. Due to abnormal anatomy, the left ureteric orifice could not be identified.

Despite the placement of a right ureteric stent, creatinine continued to rise. A nephrostomy tube was inserted the following day by interventional radiology and renal function subsequently improved.

On day three of his readmission, the rectus sheath haematoma was drained by interventional radiology. The patient recovered well following this and was subsequently discharged day thirty of readmission. Nephrostomy and ureteric stent were subsequently removed and renal function remained normal.

\section{Discussion}

There have been relevant cases reported in the literature that have been successively managed by different methods. Most these reported pelvic haematomas in the setting of massive trauma with significant pelvic fractures. Nihal et al. [2] reported a case of bilateral ureteric obstruction due to pelvic haematoma in a 23 year old male who sustained an open book pelvic fracture. Imaging demonstrated bilateral hydronephrosis and was treated with bilateral nephrostomy insertion. Hessmann et al. [3] presented three patients with pelvic haematomas secondary to pelvic fracture causing ureteric obstruction. All three patients were treated by fracture stabilisation and evacuation of haematoma. Dangle et al. [4] describe a case of spontaneous rectus sheath haematoma in an anticoagulated women that subsequently caused obstructive uropathy due to extrinsic bladder and ureteric compression. In this instance, the anticoagulant was reversed and patient underwent laparotomy and haematoma evacuation; no further decompression was required.
Significant post-operative haemorrhage in the setting of laparoscopic appendicectomy is usually due to trocar insertion, however is uncommon. This case was exacerbated by the patient being on warfarin. There are two learning points in this case. The first in making the diagnosis of obstructive uropathy, the second in treating it.

The initial cause of renal impairment was difficult to attribute to obstructive uropathy for a number of reasons. Firstly, any patient presenting with hypovolaemic shock due to haemorrhage would be expected to have a degree of renal impairment due to poor renal perfusion. Secondly, serial imaging failed to reveal radiological evidence of ureteric obstruction i.e. hydronephrosis.

Obstructive uropathy was considered when renal function continued to deteriorate despite haemorrhage control, transfusion of packed red cells and adequate fluid replacement. The patient had likely developed acute tubular necrosis due to hypovolaemia and so would be expected to have a reduced urine output which would have masked ureteric obstruction as hydronephrosis may not develop if a patient is oliguric. This diagnosis and management plan was reached after joint input from both the urology and nephrology teams. This case highlights that while hydronephrosis is usually observed in cases of ureteric obstruction, it is not always necessarily present, and absence of hydronephrosis does not exclude obstructive uropathy.

There were a number of challenges in treating this patient's obstructive uropathy once the diagnosis was made. One option was bilateral nephrostomy tube insertion. This had the advantage of avoiding general anaesthetic in an unwell patient. The disadvantage of this was that the procedure would have been technically difficult due to lack of hydronephrosis. The alternative option was ureteric stent insertion however this proved difficult in presence of distorted lower urinary tract anatomy resulting in inability to identify the left uretericorifice. In the end, the patient was treated with both modalities; this highlights that while there is no definitive evidence to suggest either percutaneous nephrostomy or retrograde ureteric stenting is a superior method of decompression of an obstructive system, the method must be decided on a case-by-case basis.

\section{Conclusion}

This case reinforces that fact that lack of hydronephrosis does not exclude ureteric obstruction. As this patient was hypovolaemic on admission, it was thought that this was the reason for his renal impairment. As it transpired, this was not the sole cause, and while it contributed to renal impairment, it also resulted in acute tubular necrosis which masked his obstructive uropathy. While the initial plan for management of his renal impairment had been bilateral retrograde ureteric stenting, anatomical distortion rendered this impossible and he was subsequently treated with unilateral retrograde ureteric stent and contralateral percutaneous nephrostomy. 


\section{References}

1. Pearle MS, Pierce HL, Miller GL, et al. Optimal method of urgent decompression of the collecting system for obstruction and infection due to ureteral calculi. J Urol. 1998;160(4):1260-4.

2. Nihal A, Fairbank AC, Palmer S, et aol. Acute bilateral ureteric obstruction complicating pelvic fracture. Injury. 1994;25(5):328-30.
3. Hessmann M, Rommens P. Bilateral ureteral obstruction and renal failure caused by massive retroperitoneal hematoma: Is there a pelvic compartment syndrome analogous to abdominal compartment syndrome? J Orthop Trauma. 1998;12(8):553-57.

4. Dangle PP, Patel MB, Terán M, et al. Obstructive uropathy secondary to rectus sheath hematoma. W V Med J. 2013;109(2):32-4.

\section{*Correspondence to:}

Nathan Grimes

MRCS, Department of Urology

Belfast City Hospital

Belfast, BT9 7AB

Northern Ireland

E-mail: nggrimes@doctors.net.uk 